\title{
Detection of pathogenic mutations in patients with non-coronary heart diseases by targeted NGS
}

\author{
D. Yermakovich ${ }^{1 *}$, L. Sivitskaya ${ }^{1}$, T. Vaikhanskaya ${ }^{2}$, A. Liaudanski ${ }^{1}$, N. Danilenko ${ }^{1}$ \\ ${ }^{1}$ Institute of Genetics and Cytology NAS, Minsk, Belarus \\ ${ }^{2}$ Republican Scientific and Practical Center of Cardiology, Minsk, Belarus \\ *e-mail: cytoplasmic@mail.ru
}

Key words: targeted NGS, non-coronary heart diseases, bioinformatics processing, pathogenic variants

Motivation and Aim: Various heart diseases, the etiology of which is not related to the change of coronary vessels, belong to the group of non-coronary. The most common of them are the autosomal dominant forms of cardiomyopathies - dilated (DCM), arrhythmogenic (AC), hypertrophic (HCM). They can develop due to a mutation in more than 100 genes. Mutations in sarcomere protein genes are the main cause of HCM and DCM [1]. Variants in genes encoding desmosomal proteins are usually detected in patients with AC [2].

Methods and Algorithms: The targeted NGS was carried out for identification of pathogenic mutations in the 24 unrelated patients with non-coronary heart diseases using the TrueSight Cardio Sequencing Kit. Additionally, investigation of the LMNA gene sequence in the 18 patients with DCM was conducted using the Nextera XT kit. The processing of raw FASTQ files was carried out using software (Trimmomatic, Bowtie2, Samtools, Bcftools, VCFlib) on the GNU/Linux platform. Using the ANNOVAR program, the primary annotation of the discovered variants was obtained. The bash script has been created to automate the processing and annotation of sequencing results. The functional impact of the variants with uncertain significance has been tested in silico by the next services: Human Splicing Finder, Jpred4, Align-GVGD, Condel.

Results: In 8 unrelated patients, 11 nucleotide variants in 8 different genes have been detected, that have a high probability of being the cause of the disease. Family genotyping showed a segregation of mutations with cardiac phenotype. In silico analysis indicated the pathogenicity of them. According to population databases, the variants have an extra low frequency $(\mathrm{MAF}<0.01)$. Four of them are described in the literature as pathogenic: rs397517853 (NEXN, nonframeshift deletion), rs267607626, rs121912496 (LMNA, missense), rs794728589 (LMNA, splicing). Two missense - rs766910280 (SCN1B), rs377473560 (MYH6) - haven't been registered as a clinical variant before. The others: stop-gain and splicing mutations in TTN and 3 missenses in MYH7, ACTC1, DES were identified for the first time.

Conclusion: The targeted sequencing is one of the most convenient and productive method of the searching for pathogenic variants in cohort of patients with polygenic diseases. In our research, the method allowed us to find 11 mutations in different genes. They have been classified as likely pathogenic and pathogenic.

\section{References}

1. Bezzina C.R., Lahrouchi N., Priori S.G. (2015) Genetics of sudden cardiac death. Current Cardiology Reports. 17(53):1-9.

2. Richard P. et al. (2003) Hypertrophic cardiomyopathy: distribution of disease genes, spectrum of mutations, and implications for a molecular diagnosis strategy. Circulation. 107:2227-2232. 